\title{
El "Mundo Uno" del presidente Arturo Frondizi y el Tratado Antártico como ejemplo de esa visión
}

María Florencia Guzmán*

\section{Resumen}

En mayo de 1958 Frondizi asume la presidencia argentina, y, desde sus inicios, planteó que la ejecución del modelo desarrollista era vital para el progreso del país. A partir de entonces, la política exterior estuvo subordinada al logro de tal objetivo, por lo que se caracterizó por poseer un tinte pragmático, a pesar del contexto internacional que primaba en el momento, es decir, la Guerra Fría. Por tal motivo, a lo largo de su gestión mantuvo relaciones diplomáticas y comerciales con diferentes países, sin distinción de ideología, ya fueran capitalistas o comunistas. Frondizi aseguraba que en el futuro nos encontraríamos en un mundo completamente globalizado, desarmado y desnuclearizado, al que llamaba "Mundo Uno". En consecuencia, la firma del Tratado Antártico puede ser entendida como un momento representativo de tal pensamiento. Argentina se convierte en un actor fundacional del régimen antártico donde la cooperación científica y las relaciones pacíficas entre los países es uno de sus rasgos distintivos.

Palabras claves: Frondizi - política exterior argentina - Tratado Antártico

\section{President Arturo Frondizi's "One Single World" and the Antarctic Treaty as an example within his vision}

\begin{abstract}
Frondizi assumed the Argentine presidency in May 1958, and, from the beginning, stated that the implementation of a development model was vital for the country progress. From then on, foreign policy was subordinated to achieving this objective, which is why it was characterized as having a pragmatic orientation, despite the international context that prevailed at the time, the Cold War. For this reason as well, throughout his tenure he maintained diplomatic and commercial relations with different countries, without distinction of ideology, whether capitalist or communist. Frondizi assured that in the future we would find ourselves in a completely globalized, disarmed and denuclearized world, which he called "One Single World". The Antarctic Treaty can be understood as a sample of such thinking. Argentina became a founder country of the Antarctic regime, whereas the scientific cooperation and peace among countries is one of its distinctive features.
\end{abstract}

Key words: Frondizi - Argentina’s foreign policy - Antarctic Treaty

TRABAJO RECIBIDO: 12/05/2020 TRABAJO ACEPTADO: 02/09/2020

Esta obra está bajo una licencia internacional https://creativecommons.org/licenses/by-sa/4.0/

\footnotetext{
* Licenciada en Relaciones Internacionales, Facultad de Ciencia Política y Relaciones Internacionales, Universidad Nacional de Rosario (UNR, Argentina). Maestranda en Integración y Cooperación Internacional (CERIR-CEI-UNR). Correo electrónico: florguzmanc2@gmail.com
} 


\section{Introducción}

Arturo Frondizi asume su mando presidencial en un período convulso, tanto a nivel nacional como en el plano internacional. En lo interno, el entonces presidente se encontraba vigilado por diferentes sectores de la sociedad civil, sobre todo fuerzas armadas, sindicalistas y peronistas. En lo que respecta a la situación internacional, el mundo se encontraba transitando la Guerra Fría, la cual "surgió de la bipolaridad política e ideológica en que quedó dividido el mundo al finalizar la Segunda Guerra Mundial y de la competencia de los dos bloques mundiales por el control estratégico de las áreas geográficas" (Leal Buitrago, 2003, p.77).

En dicho contexto es que el mandatario intentó llevar a cabo una política exterior al servicio de su estrategia nacional desarrollista y de integración, y por tal motivo es que la misma se caracterizó por ser anti-ideológica y pragmática, con tintes de cierto idealismo cosmopolita.

La firma del Tratado Antártico el 1 de diciembre de 1959, en donde Argentina participó de la redacción del mismo como miembro fundador del Régimen Antártico, fue un gran acontecimiento que marcó dicha política, dado que usualmente el país acostumbraba a ser firmante de acuerdos que ya se encontraban redactados y establecidos; por eso, este motivo es uno de los tantos que remarca la importancia de este Tratado para el país. A su vez, dicha firma es un reflejo íntegro de la política exterior del ex mandatario y su visión del "Mundo Uno"1, caracterizado por la desnuclearización y por encontrarse totalmente globalizado, sin ningún tipo de división que dificulte las relaciones entre diferentes Estados. Por esta razón, considero relevante otorgar un lugar destacado a la firma del Tratado Antártico en este trabajo (Ministerio de Relaciones Exteriores, Comercio Internacional y Culto, s/f).

Es por ello que la presente contribución parte del supuesto de que la política exterior de A. Frondizi, si bien se caracterizó por estar subordinada a sus objetivos desarrollistas y poseer un tinte anti-ideológico (no se encontraba influenciada por las ideas que predominaban en el mundo en aquel entonces, específicamente, la estricta división entre el capitalismo y el comunismo), también estuvo signada por la voluntad de promover una mayor integración y relaciones multilaterales, además de abogar por los principios de no intervención, autodeterminación de los pueblos, así como también de ser promotora de la paz².

A partir de esto, el objetivo principal de este trabajo será analizar la política exterior llevada a cabo por la presidencia de Arturo Frondizi entre los años 1958 y 1962, haciendo hincapié en la firma del Tratado Antártico de 1959 (Casa Rosada, s/f).

En consecuencia, los objetivos específicos serán describir tanto el contexto interno como externo en el cual el presidente argentino ejerció su mandato, analizar los lineamientos de la política exterior que llevó a cabo durante su mandato presidencial y, por último, explicar el contexto de la firma del Tratado Antártico, como uno de los significativos ejemplos de la política exterior de Frondizi en su búsqueda de un mundo desarmado.

\footnotetext{
${ }^{1}$ El concepto de "Mundo Uno" será explicado más adelante en el presente trabajo.

${ }^{2}$ En gran medida, seguimos las consideraciones que hacen autores como Cardone (2013) en su libro titulado Porque Frondizi fue un estadista, quien estudia su biografía, centrándose en las principales acciones llevadas a cabo por el ex mandatario durante su gestión. Allí, no sólo hace hincapié en los hechos más relevantes de su mandato, tanto a nivel interno como internacional, sino que también analiza las posturas adoptadas por Frondizi frente a estos y sus fundamentos. Continuando con esta línea, Llairó y Siepe (2003), en el libro "Frondizi, un nuevo modelo de inserción internacional", contribuyen con un análisis histórico de la gestión de Frondizi destacando su política exterior y, en ese marco, explican tanto el contexto interno e internacional de la gestión del ex mandatario, como así también los factores que condicionaron a su accionar.
} 


\section{Frondizi en la presidencia: su política exterior en un contexto interno e internacional convulso}

A fin de comprender la presidencia de A. Frondizi y su llegada al poder el 1 de mayo de 1958, tal como indica uno de los objetivos específicos resulta imprescindible describir tanto el contexto interno como el internacional que se imponía al momento de su elección presidencial, como también a lo largo de los 4 años que dura su mandato (Casa Rosada, s/f).

En cuanto al contexto internacional, el mandatario argentino inicia, transcurre y finaliza su presidencia en un periodo histórico conocido como Guerra Fría. Dicho escenario comienza con el fin de la Segunda Guerra Mundial, cuando las dos superpotencias, Estados Unidos y la Unión Soviética, intentan establecer el nuevo orden mundial bajo sistemas que ambas defienden: el capitalismo y el comunismo. Esto generó una feroz rivalidad, debido a que estas potencias buscaban que uno de estos dos sistemas prevaleciera por sobre el otro, dando así inicio al período mundialmente conocido como bipolar, que llegaría a su fin en el año 1989 con la caída del muro de Berlín para algunos investigadores, o en 1991 con la implosión de la Unión Soviética para otros (McMahon, 2009).

Durante este período, que duró décadas, el mundo quedó dividido en dos, gran parte se encontraba bajo el orden capitalista propio de los Estados Unidos, mientras que otra parte se mantenía en la órbita comunista bajo el ala de la entonces llamada Unión Soviética. Esta división ideológica también puede ser explicada y comprendida por diferentes académicos en términos realistas, puesto que primaba la lógica de balance de poder junto con las cuestiones de seguridad ${ }^{3}$.

Sin embargo, el conflicto no era directo entre Estados Unidos y la Unión Soviética, dado que la estrategia era atraer a terceros países a sus respectivas zonas de influencia, ya sea por medio de alianzas político-militares como a través de relaciones económicas. Y, si bien en un comienzo el escenario fue la Europa de la posguerra, con el inicio de la guerra de Corea en 1950 "se trasladó desde el centro de la política internacional a la periferia" (McMahon, 2009, p. 99). Es decir, a partir de ese momento, la Guerra Fría adquiere un carácter totalizador, "pocos rincones del mundo consiguieron mantenerse ajenos a la red de rivalidad, competición y conflicto en que se hallaban atrapadas las superpotencias" (McMahon, 2009, p. 98).

A pesar de que en un principio las superpotencias querían evitar todo enfrentamiento militar directo entre ambas, la Guerra Fría supuso una carrera armamentística sin precedentes, ya que la misma adquirió el carácter único de la dimensión nuclear con la destrucción mutua asegurada. Tal como afirmó Cohen (1993) en McMahon (2009, p. 93), "lo que era una rivalidad política sistémica pasó a ser una confrontación militarizada que obedecía a motivos ideológicos y suponía una amenaza para la supervivencia del planeta”.

Argentina no fue ajena a lo ocurrido en aquella época. El mundo al estar tan interconectado no le permitía serlo. Por estar el país geográficamente ubicado en el lado occidental (continente americano), se pretendía que siguiera los parámetros y reglas impuestas por el capitalismo impartido desde los Estados Unidos, eliminado así todo tipo de relación, sea diplomática o comercial, con el bloque soviético.

No obstante, al contrario de lo que el bloque capitalista esperaba del gobierno de Argentina en cuanto a sus relaciones diplomáticas y comerciales, durante de la presidencia de Frondizi, Estados Unidos no pudo hallar a un presidente sumiso que acatara con exactitud las normas que imperaban en el sistema bipolar.

Frondizi, para inquietud de la potencia capitalista y sus seguidores, fue fiel al pragmatismo que tanto caracterizó a su gobierno. Durante su mandato, se mantuvo firme y constante con su pensamiento, ya que éste era imprescindible para promover una política exterior que fuera útil al desarrollismo que pretendía instaurar en el país, por lo que el sesgo ideológico

\footnotetext{
3 "Las cuestiones internacionales se leían, casi exclusivamente, desde la óptica de la seguridad y ninguna de las dos Superpotencias estaba dispuesta a ceder o perder respecto de la otra en el balance de poder e influencia" (Colacrai, 2001, p. 41).
} 
impuesto para Occidente no fue un límite para el presidente a la hora de entablar relaciones con diferentes países.

A partir de lo dicho, resulta importante explicar que la línea de pensamiento desarrollista, impulsada desde la CEPAL ${ }^{4}$, comienza a cobrar relevancia en la Argentina en la década de 1950, como un plan de modelo político y económico a seguir a fin de implementarlo en el país, en donde la iniciativa privada y los capitales extranjeros son elementos dinamizadores a destacar, ya que son los principales conductores del crecimiento de un Estado. Entre los seguidores de este pensamiento se encontraban intelectuales e investigadores de diferentes sectores políticos, siendo Rogelio Frigerio el principal ideólogo, y, además, quien apoyó y acompañó la candidatura de Frondizi a la presidencia argentina en el año 1958 (García Bossio, 2012, p.5).

Con relación al contexto interno, el mismo puede describirse como delicado, dado que luego del golpe de Estado cívico-militar de 1955, históricamente conocido como la Revolución Libertadora, el radicalismo era el principal partido no peronista candidato a ganar las elecciones presidenciales de 1958. El liderazgo dentro de la Unión Cívica Radical (UCR) para la conducción del país se lo disputaban entre Ricardo Balbín y Frondizi, pero al momento en que este último gana las internas, el partido se dividió en dos: Unión Cívica Radical Intransigente (con Frondizi como líder) y Unión Cívica Radical del Pueblo (Ricardo Balbín como representante) (Szusterman, 1993).

La principal diferencia entre ambos bloques residía en la posición que cada sector asumió frente al peronismo (partido proscripto luego de la Revolución Libertadora), aunque al momento de gobernar, tanto líderes de la Unión Cívica Radical Intransigente (UCRI), como de la Unión Cívica Radical del Pueblo (UCRP), levantarían la proscripción al peronismo, a pesar de cuál había sido su postura en un inicio frente a este hecho.

La UCRP de Balbín, era la gran oposición al partido peronista, y su apoyo provenía esencialmente de los militares, la Iglesia y otros sectores más conservadores de la sociedad civil, como por ejemplo el agroexportador; mientras que la UCRI de Frondizi, que reivindicaba al nacionalismo de Yrigoyen, se caracterizaba por su cercanía al peronismo en cierta manera, ya que creía firmemente que la solución era formar un gran frente nacional, teniendo una actitud conciliadora (Espacio Radical, 2019).

Al momento de las elecciones, dentro de la UCR, la UCRP fue la más votada; sin embargo, éstas fueron anuladas ya que habían sido denunciadas por ilegales por ser convocadas por los militares. En la segunda vuelta, consecuencia de un nuevo llamado a elecciones, la UCRI ganó y Frondizi asumió a la presidencia el 1 de mayo de 1958 (Espacio Radical, 2019).

Estas elecciones fueron un tanto polémicas, a causa de que Frondizi había firmado un pacto con el partido peronista, con el objetivo de recibir votos y apoyo de su electorado, a cambio de poner punto final a la proscripción del peronismo que había en el país en ese entonces. Este acuerdo, si bien le permitió llegar a la presidencia de la Nación Argentina, también produjo ciertas crisis institucionales desde un primer comienzo, ya que su gestión comenzó con cuestionamientos acerca de la legitimidad y la representatividad del gobierno.

Sin embargo, ésta no fue la única controversia del entonces presidente durante los años de su administración, sino que a lo largo de la misma tuvo diferentes conflictos que hicieron peligrar su presidencia en varias ocasiones, ya que su gestión, poco ortodoxa y, sobre todo, muy poco convencional para la época, le fue restando apoyo en diferentes sectores de la sociedad. Tal como afirma Potash (1986) citado en Colacrai (2002, p. 55), "sólo merced a su pertinaz

\footnotetext{
${ }^{4}$ La CEPAL, en su documento Desarrollo e Integración en América Latina (2016, p. 290), explica lo siguiente: "A partir de los años cincuenta, el debate sobre el Estado y la economía se ve influenciado por las ideas desarrollistas impulsadas desde la CEPAL. Raúl Prebisch, Celso Furtado, Medina Echeverría y Aníbal Pinto se cuentan entre los intelectuales que señalaron la necesidad de la acción del Estado en el impulso de políticas industriales destinadas a superar la dependencia y la asimetría de los términos de intercambio que caracterizaba la inserción económica de América Latina en el mundo".
} 
determinación de mantenerse en la presidencia pudo manejar con habilidad la gran oposición que tenía en el Ejército, mientras gozaba de cierto apoyo de la Marina”.

Por un lado, su política desarrollista aparejada con las intenciones de modernizar al país, fomentando a la utilización de nuevas tecnologías e inversiones extranjeras de capital, generó grandes tensiones, sobre todo en aquellos sectores que se resisten a generar un cambio dentro de la estructura agroexportadora. El principal objetivo de Frondizi era integrar a la industria y al sector del agro, a partir de transformaciones tecnológicas que serían llevadas a cabo gracias a la implementación del modelo desarrollista.

El modelo desarrollista partía de la base que el país estaba subdesarrollado y que la economía dependía de la importación de insumos y bienes de capital necesarios para la industria, que era básicamente liviana. Para poder realizar estas importaciones se necesitaban divisas que provenían principalmente de las exportaciones de productos agropecuarios (Blanco, 2015, p.14).

Empero, los conflictos que acentuaban la crisis institucional no provenían únicamente de parte de estos sectores, es decir, los agroexportadores, y sus representantes no eran los únicos opositores. Las fuerzas armadas, por ejemplo, llevaron a cabo seis intentos de golpe militar a lo largo de su mandato. Uno de los grandes motivos se debió a la cercanía que Frondizi tenía con el peronismo, sobre todo, en el breve período en que eliminó la proscripción del partido y éste pudo prevalecer en las elecciones provinciales. Como consecuencia de esto, el entonces presidente proscribió nuevamente al partido, y las fuerzas armadas intervinieron las provincias, ejecutando el Plan Conintes.

El 14 de noviembre de 1958, el presidente aprobó un Decreto secreto -9880- que ponía en marcha dicho Plan, que se caracterizaba por "disponer el establecimiento de un sistema de zonas, subzonas y áreas de defensa bajo autoridad militar" (Pontiero, 2015, p.7), y, el cual autorizaba a las fuerzas armadas a reprimir protestas y cualquier movimiento subversivo.

Estas manifestaciones en mayoría eran llevadas a cabo por sindicalistas descontentos con Frondizi y las políticas económicas que llevaba a cabo (se oponían fervientemente a la política desarrollista, tanto a nivel económico como enmarcada dentro de la política exterior) y por mantener la proscripción al peronismo, a pesar del pacto que había hecho con el partido de eliminarla, y gracias al cual, en parte, pudo salir triunfante en las elecciones presidenciales en el año 1958 (la misma fue eliminada por un breve lapso). Por tales motivos, el presidente también se encontraba en situación de conflicto con el peronismo y sus seguidores.

El acercamiento con Cuba durante la revolución tampoco fue de agrado para los sectores más conservadores de la sociedad, como los militares y la Iglesia que reaccionaron frente a este hecho y acusaron de comunista a Frondizi.

Por último, los radicales tampoco aprobaban la gestión, no sólo por la cercanía con el peronismo, sino también por el modelo desarrollista que pretendió implementar en el país, con la apertura al capital extranjero e inversiones, sobre todo, las que estaban relacionadas con cuestiones petroleras no fueron bien aceptadas.

Para comprender la postura que adoptó Frondizi frente a la cuestión de la apertura al capital extranjero, resulta imprescindible destacar que al momento en que asume la presidencia, el contexto nacional se caracterizaba por tener una balanza comercial deficitaria, un estancamiento en la producción, desprotección total de la industria argentina y, un sistema energético completamente ineficiente. Por tales motivos, se requerían inversiones extranjeras urgentemente.

A partir de estos hechos, su programa desarrollista estaba basado en el progreso de la industria nacional en la totalidad de sus sectores, pero destacando al petróleo, la siderúrgica, el carbón, la energía eléctrica, la petroquímica, el gas y la celulosa (Blanco, 2015, p. 15).

Sin embargo, la cuestión del petróleo fue primordial en su gestión, lo que suscitó conflictos con diferentes sectores, sobre todo con el radicalismo, tal como se mencionó 
anteriormente. El objetivo de Frondizi era lograr el autoabastecimiento en este sector, para dejar de importar progresivamente, y poder disponer de ese dinero para la compra de maquinarias y tecnologías que otros países podían ofrecernos.

Para lograr tal cometido, se precisaba indiscutiblemente de inversiones extranjeras, por lo que, en consecuencia, aprobaron dos leyes en diciembre de 1958, la ley 14780 y la 14781 . En cuanto a la primera de estas, dispuso "equiparar los capitales extranjeros a los nacionales, cuando las inversiones se dediquen a la promoción de nuevas actividades productivas, o a la ampliación y/o perfeccionamiento de las existentes" (Frigerio en De Pablo, 1998, p.2).

En consecuencia, con el objetivo de revertir la situación en la que el país producía únicamente el 30\% del petróleo e importaba el resto, y alcanzar el autoabastecimiento, se firmaron contratos con compañías extranjeras; la mayoría eran concesiones a empresas de origen estadounidense, para extraer el crudo y vendérselo a YPF.

Como resultado de esta política, Frondizi logró alcanzar en cierta medida su objetivo en dicho sector, ya que:

La producción total pasó de 5,7 millones de metros cúbicos en 1958, a 15,6 millones en 1962, lo cual implica un aumento de $175 \%$, en tanto que la importación cayó de 7,5 millones de metros cúbicos en 1958 (57\% del consumo total) a 1,2 millones en 1962 (7\% del consumo total). Aunque menos que la de los contratistas, entre 1958 y 1962 también aumento la producción propia de YPF (110\%), la cual en 1962 alcanzó a 2/3 de la producción total (De Pablo, 1998, p. 7).

Sin embargo, tal como se mencionó, diversos sectores políticos en el país se oponían a estas medidas, especialmente a que el petróleo fuera extraído con la ayuda de capitales extranjeros; alegaban que podía realizarse la extracción del crudo de forma más dinámica y ventajosa con la mera participación del Estado Nacional. Empero, estos sectores no tenían en cuenta ciertas limitaciones que el país tenía, tanto en cuestiones técnicas como financieras.

Cómo puede apreciarse, la llegada de A. Frondizi al poder, junto con su visión desarrollista en consonancia con una política exterior de tinte pragmático, generó ciertas rispideces tanto con la potencia capitalista dominante, Estados Unidos, como con ciertos sectores de la sociedad civil argentina, tal como fueron explicados brevemente con anterioridad.

R. Putnam analizó que los Estados y sus políticas exteriores a nivel interno, se encuentran influenciadas por ciertos actores sociales y grupos de interés que pretenden obtener beneficios, y a su vez, a nivel internacional, permanecen influencias por Estados o instituciones internacionales que pretenden lograr ciertas ventajas por medio de negociaciones o tratados internacionales, por ejemplo (Putnam, 1996).

Frondizi, durante toda su gestión tuvo conflictos y crisis institucionales, pese a sus esfuerzos de llevar adelante negociaciones de doble nivel, tal como explica R. Putnam.

Con el propósito de hacer un análisis de su política exterior, tal como se plantea en uno de los objetivos específicos, corresponde dar cuenta del mundo "globalizado" al momento en el que llega al poder. Por tal motivo, es importante mencionar hechos que ocurrían a nivel internacional durante su gestión, como, por ejemplo, la formación de Comunidad Económica Europea y la emergencia de Japón como una incipiente potencia, enmarcado en un contexto de Guerra Fría. A su vez, "cuando Frondizi asumió la presidencia de la Argentina, la economía del mundo occidental y el comercio internacional crecían a una alta tasa, promovidos por los procesos de cooperación e integración económica" (Blanco, 2015, p.16).

Es entonces que, analizando estos acontecimientos, decide mejorar la inserción de Argentina en el mundo por venir, que él entendía como "Mundo Uno". Se oponía firmemente a que nuestro país quedase por fuera del sistema internacional que se aproximaba, por lo que la integración en el mundo globalizado debía realizarse de forma pragmática, sin tener en cuenta las ideologías que prevalecían en su momento. Por esta razón es que se reserva la conducción de las relaciones internacionales, instruyendo a sus funcionarios electos (Cardone, 2013). 
Asimismo, "el presidente anhelaba para la Argentina un mayor protagonismo en las decisiones del bloque occidental y una suerte de representación oficiosa de las pretensiones latinoamericanas frente Estados Unidos y Europa" (Machinandiarena de Devoto, 2015, p. 209).

Teniendo en cuenta este futuro mundo globalizado, las relaciones que mantenía con el mercado y el capital extranjero, se caracterizaron por ser sumamente cordiales, ya que, para implementar el modelo desarrollista en nuestro país, se necesitaba de estos dos.

Frondizi, como diputado primero y como presidente después, siempre sostuvo la necesidad de mantener una política de intercambio con todos los países del mundo; en las medidas de sus posibilidades, esa tendencia la mantuvo durante todo su período de gobierno. Pero permanentemente existieron presiones movidas por intereses ideológicos y económicos, tanto de carácter nacional como internacional (Llairó y Siepe, 2003, p. 149).

Es así que pone la política exterior al servicio del desarrollo, debido a que precisaba de capitales externos e inversiones para la infraestructura y generar una estructura productiva, a fin de crear un modelo de capitalismo industrial moderno, y a pesar de las controversias que esto generó con diferentes sectores políticos de la sociedad argentina, tal como puede apreciarse en la explicación que se otorgó en párrafos anteriores con la cuestión petrolera.

Como dependía mayoritariamente de dicho capital para la consecuente implementación del modelo desarrollista, no debía formar parte del conflicto de la Guerra Fría, a fin de fomentar relaciones de negocios con muchos países, independientemente de la brecha este-oeste, y atrayendo el mayor capital posible.

Prueba de este pensamiento fue expresado en su primer discurso como presidente, en donde remarca la importancia de una política exterior sin ideología y conservando el principio de no intervención: "Argentina deberá comerciar con todas las naciones de la tierra sin discriminación y sin inmiscuirse en los problemas internos de otros países" (Fundación Frondizi, 2014, p.46).

El mandatario realizó visitas destinadas a fomentar el comercio e intercambio con numerosos países a lo largo de su gestión, con el objetivo de explicar su política desarrollista y mostrar a la Argentina como un país industrial. Con el objetivo de obtener inversiones extranjeras directas, creó un marco jurídico adecuado para facilitar su entrada al país. "En algo Frondizi fue muy preciso: no aceptaba que las fronteras ideológicas afectarán, por un lado, la apertura comercial, y por el otro, nuestra posición y rango en el mundo, es decir nuestra capacidad de negociación" (Llairó y Siepe, 2003, p. 15).

Claros ejemplos de este tipo de vínculos comerciales sin sesgo ideológico son los acuerdos comerciales y financieros llevados a cabo con la Unión Soviética en 1958, por los cuales esta última daba un crédito a la Argentina para abastecerse de suministros y equipamientos destinados a la industria petrolera, y a cambio de esto la Unión Soviética se cobraba en mercadería argentina (Llairó y Siepe, 2003, p. 145).

Sin embargo, debido a las constantes presiones que el gobierno recibía por comerciar con países del este, el intercambio fue restringiéndose, y en el año 1961, la Argentina representaba un total de un $1 \%$ del comercio en el espacio socialista (Llairó y Siepe, 2003).

En definitiva, tal como aseguran en su libro Llairó y Siepe (2003):

Lo que dificultó esta ampliación del comercio con la Unión Soviética, especialmente en lo que se refería a importación fue, en primer lugar, las discriminaciones de todo tipo, y en segundo lugar, los temores que despertaba el comercio con la URSS por lo antedicho y por la presión de círculos tanto oficiales como extranjeros que trataban de disuadir a quienes, a pesar de todo, se hallaban dispuestos a seguir con este comercio (p. 147).

Empero, "en los primeros años Frondizi dio prioridad a las relaciones económicas con Estados Unidos en virtud de que este país era la principal fuente disponible de capitales para instrumentar los programas de desarrollo argentino" (Machinandiarena de Devoto, 2015, p. 209). 
Es por ello que resulta importante destacar las relaciones que el presidente mantuvo con Estados Unidos, las cuales involucran parte de la gestión de los mandatarios estadounidenses Eisenhower y Kennedy.

Desde el momento en que asumió la presidencia, Frondizi entendía que era imprescindible mantener relaciones sumamente cordiales y cercanas con tal país. Consideraba que las mismas eran esenciales para emprender el camino del modelo desarrollista, ya que catalogaba a aquel país como proveedor clave de tecnologías, inversiones directas y desarrollo industrial. Tal como se explicó, Argentina afrontaba graves problemas económicos, y esperaba que Estados Unidos pudiera ayudar a revertir dicha situación.

El presidente, así lo manifestó en uno de sus discursos:

El destino de América Latina está en manos de los doscientos millones de habitantes; según cual sea la política que desarrollen los Estados Unidos en relación con nuestra América, nuestro camino hacia la libertad, el desarrollo y la justicia será más o menos doloroso, más o menos largo (Frondizi citado en Llairó y Siepe, 2003, p. 51).

Tal era la importancia que le asignaba a las relaciones con el país del norte, que fue el primer presidente argentino en realizar visitas oficiales a los Estados Unidos. A lo largo de su mandato, realizó un total de tres viajes presidenciales, manteniendo conversaciones con los presidentes norteamericanos Eisenhower y Kennedy ${ }^{5}$.

Sostenía que dicho viaje era un punto esencial para sellar la orientación que había otorgado a su política exterior desde su asunción a la presidencia el $1^{\circ}$ de mayo de 1958 , ya que, como se explicó a lo largo del presente trabajo de investigación, su política exterior se encontraba al servicio de su gran objetivo de implementar el modelo desarrollista en nuestro país.

En relación a lo dicho, Devoto y Pelosi (2009) señalaron lo siguiente:

La necesidad de fortalecer la ubicación de la Argentina en el concierto internacional y de contribuir, a través de ello, al desarrollo nacional habían inspirado la decisión de traducir en los hechos el reconocimiento de la posición americana y occidental que correspondía a la Argentina (p. 69).

En estos viajes oficiales, Frondizi planteaba sobre todo la cuestión de la relación entre el subdesarrollo en las naciones latinoamericanas con el consecuente peligro del comunismo, debido a que, para el entonces presidente argentino, la falta de desarrollo en el continente traería aparejadas posibles guerrillas comunistas, es decir que los atrasos en cuestiones económicas terminarían desembocando en problemas de seguridad hemisférica.

Durante su primera visita, el 21 de enero de 1959, planteó lo siguiente en su discurso ante el Congreso de Estados Unidos en la ciudad de Washington:

A vosotros no puede seros indiferente que haya millones de individuos que vivan mal en el continente americano. La condición de esos semejantes es no solamente una apelación a nuestros ideales comunes de solidaridad humana, sino también una fuente de peligro para la seguridad del hemisferio. Dejar en el estancamiento un país americano es tan peligroso corno el ataque que pueda provenir de una potencia extracontinental (Discurso de Frondizi en Lanús, 2000, p. 201).

Su férrea posición frente a la imperiosa necesidad de implementar el modelo desarrollista se observa en la posición que el mismo toma frente a la Alianza para el Progreso, propuesta por Kennedy en marzo de 1961 (Morgenfeld, 2001, p.141).

\footnotetext{
${ }^{5}$ Su primer viaje constó de la siguiente agenda: "permaneció en ese país desde el 19 de enero hasta el 1 de febrero de 1959. La visita de estado a Washington tuvo lugar entre el 20 y el 23 de enero, y el presidente luego llevó a cabo actividades en Chicago, Detroit, Nueva York y Miami” (Devoto y Pelosi, 2009, p. 69).
} 
El entonces presidente norteamericano planteó un programa de asistencia en cooperación técnica, económica y social destinado a Estados latinoamericanos, bien conocido como Alianza para el Progreso. Empero, éste tenía la condición de que cada uno implementase el desarrollo por cuenta propia. En verdad, el trasfondo de la misma era la lucha contra el riesgo comunista en el continente.

Frondizi se mantuvo en desacuerdo con la propuesta del programa de Kennedy, ya que alegaba que el esquema era meramente asistencialista, y lo que realmente precisaba América Latina era una cooperación que estuviese vinculada sobre todo a cuestiones de desarrollo básico, como por ejemplo la infraestructura, el transporte, la energía, entre otros.

La relación que el entonces presidente mantuvo con los Estados Unidos, se caracterizó por estar enmarcada dentro de su política exterior de tinte pragmático y estratégico, ya que, como pudo observarse, estuvo signada en las cuestiones en torno a la implementación del modelo desarrollista en nuestro país, y por su ferviente convicción de que, a través de la implementación de dicho modelo, el progreso sería netamente inevitable a mediano plazo.

Sin embargo, Frondizi entendía los límites económicos y financieros que suponía abocarse únicamente al mercado estadounidense, lo que explica el gran objetivo de buscar nuevos mercados, en consonancia con la imperiosa necesidad de atraer posibles inversiones al país y establecer relaciones comerciales, lo cual era clave, tal como se explicó con anterioridad, para el programa desarrollista que deseaba implementar en Argentina. Por tal motivo, se pueden comprender también, las siguientes relaciones bilaterales que estableció a lo largo de su gestión con diferentes países.

En noviembre de 1961, el presidente realizó una visita oficial a Canadá, convirtiéndose de esta manera en el primer mandatario latinoamericano que arribaba a dicho país en un viaje de índole diplomático. Era de notorio y público conocimiento que ambos países (Argentina y Canadá) tenían semejanzas en lo que respecta a sus actividades económicas como en su extensión geográfica, por lo que nunca se esperó grandes posibilidades de intercambio con tal nación. (Devoto, 2010)

Sin embargo, con la visita de Frondizi fue factible el arribar a un acuerdo: "en Ottawa se firmó un convenio entre la Argentina y Canadá por el cual este país vendía 70 locomotoras diesel eléctricas, valuadas aproximadamente en 14 millones de dólares, por la firma Montreal Locomotive Works Limited" (Devoto, 2010, p. 151). De todas formas, esto se hizo posible gracias a la amplia financiación que otorgó el gobierno canadiense.

El presidente también realizó visitas oficiales a Europa entre junio y julio de 1961. Su agenda abarcó un total de 9 países europeos y, los motivos principales de tal viaje fueron dos: "fortalecer las relaciones con los tradicionales socios europeos y prevenir, en la medida de lo posible, las tendencias restrictivas que se insinuaban" (Machinandiarena de Devoto, 2015, p. 219), como consecuencia de la conformación del Mercado Común Europeo, que buscaba desarrollar la producción de materia prima y alimento, lo cual implicaba ciertas políticas proteccionistas hacia dicho sector, buscando obtener como resultado una significativa reducción de las importaciones en dicha área, perjudicando a toda la Argentina, además de al resto de Latinoamérica. El desarrollismo se tornaba cada vez más necesario y significante.

Con motivo de dicha gira presidencial, el gobierno británico le extendió la invitación para que fuera al Reino Unido, ya que Argentina era, en ese entonces, su socio comercial de mayor importancia en América Latina. Frondizi aceptó cordialmente la invitación; su gran propósito consistía en obtener créditos a largo plazo e inversiones británicas. Si bien no pudo lograr en gran medida los objetivos que pretendía, especialmente la cuestión de la asistencia financiera en grandes proporciones, la visita al Reino Unido puede considerarse un gran éxito por parte del presidente argentino, debido a que "logró un cambio radical en la percepción británica de la situación de la Argentina que no ocurría desde muchos años atrás" (Machinandiarena de Devoto, 2015, p. 207). 
Otra de las visitas oficiales tuvo como destino a España, país con el que se habían tensado las relaciones desde la primera década de 1950 como resultado de rumores que afirmaban que Perón estaba dispuesto a romper todo tipo de vínculos con el gobierno franquista. Por tal motivo, la llegada a España fue considerablemente significativa.

Gracias a que tanto los intereses culturales como económicos fueron invocados, y que se realizaron grandes esfuerzos en dejar de lado las diferencias ideológicas, haciendo hincapié en el eje en común de la persecución del desarrollismo "los objetivos de la política exterior del país sudamericano y del tardofranquismo volvieron a coincidir" (Carsen, 2017, p. 3).

El resultado de las reuniones fue positivo, puesto que se firmó un Convenio de Migración entre los dos países. El mismo contempla, resumidamente, los siguientes puntos, según la Organización Internacional del Trabajo (s/f):

Los inmigrantes españoles tienen los mismos derechos y obligaciones que los argentinos en todo lo que concierne a la remuneración, condiciones de trabajo y seguros sociales. Reglamenta las modalidades del proceso migratorio en ambos países, desde el reclutamiento en España hasta el visado consular de entrada a la República Argentina. Establece operaciones colectivas (entre España y Argentina) referentes especialmente al fomento y desarrollo en Argentina de colonizaciones agropecuarias, explotaciones industriales, pesqueras, forestales y mineras.

Un comunicado oficial por parte de ambos mandatarios expresaba la siguiente conclusión del viaje oficial de Frondizi: "se ha destacado la necesidad de que ambos Gobiernos intensifiquen, dentro de sus respectivas posibilidades, los esfuerzos destinados a lograr una más adecuada coordinación y cooperación económica" (ABC, 1960, citado en Carsen, 2017, p. 11).

Por otra parte, la agenda del presidente argentino en torno a las visitas oficiales con el propósito de concretar acuerdos económicos y comerciales de tipo bilateral que fueran útiles a su objetivo desarrollista, se expandió a distintos países asiáticos. Gran ejemplo de ello son los viajes realizados a Tailandia y Japón en el año 1961.

Frondizi fue el primer mandatario latinoamericano en realizar un viaje oficial a Tailandia ${ }^{6}$. Merced a dicha visita, Tailandia y Argentina llegaron a un acuerdo en diciembre de 1961, el cual tenía como objetivo principal, aumentar el volumen del intercambio comercial entre ambos países y, promover las relaciones económicas. Adicionalmente, "en noventa días se comprometían a suministrar recíprocamente las listas de materias primas y mercaderías a exportar. En cuanto a derechos de aduana e impuestos de exportación e importación se otorgarían un tratamiento favorable y de no discriminación" (Devoto, 2010, p. 163).

En Japón, Frondizi también fue el primer mandatario latinoamericano en realizar una visita presidencial. La misma se concretó entre el 13 y 21 de noviembre del año 1961. Resulta trascendente hacer mención de que nuestro país necesitaba en aquel entonces de maquinarias y bienes de capital para implementar el modelo desarrollista, y que a su vez, Argentina le exportaba carnes, cueros, lanas y granos principalmente, y que, debido al gran dinamismo que el país asiático tenía, se podía esperar a que se convirtiera en un futuro gran importador de nuestras materias primas; por ello, la importancia del viaje oficial ${ }^{7}$ (Devoto, 2010).

Finalmente, como resultado de la visita oficial del presidente argentino a Japón, ambos países firmaron un Tratado de Amistad, Comercio y Navegación (1961) que deba por establecido lo siguiente:

\footnotetext{
6 El mismo se concretó “entre el 8 y el 11 de diciembre de 1961. Junto a su esposa fueron huéspedes del rey Bhumibol Adulyadej y la reina Sirikit” (Devoto, 2010, p. 157).

${ }^{7}$ En palabras del propio Frondizi, citado en Devoto (2010): “Japón había sabido dar al mundo el ejemplo invalorable de que es perfectamente compatible la rápida evolución social y las profundas transformaciones en el orden político, económico y cultural, con el respeto por lo tradicional y por lo mejor de la herencia de las generaciones pasadas" (p. 171).
} 
"Firme y perpetua paz" entre los dos países y sus pueblos, y acordaba recíprocamente el tratamiento nacional y de nación más favorecida. Ambas partes se comprometían a expandir el comercio, fortalecer las relaciones económicas y fomentar el intercambio de conocimiento científico y técnico. Por canje de notas se acordó, además, que ambas partes tratarían de adecuar su comercio e intercambio a prácticas justas internacionalmente aceptadas y cooperarían para impedir las perjudiciales (Devoto, 2010, p. 172).

En cuanto a su visión de política exterior respecto a Latinoamérica, Frondizi promovía una mayor integración de los Estados latinoamericanos. Su objetivo era proyectar una política económica común, a fin de evitar el deterioro de vida de la población, resultado de un modelo basado únicamente en la extracción de materias primas. Además, pretendía fomentar las relaciones comerciales entre los países vecinos y promover el principio de no intervención en los asuntos internos de los latinoamericanos.

Esta línea de pensamiento integracionista se puede encontrar en sus discursos presidenciales: "Una acción conjunta de las naciones latinoamericanas puede influir decisivamente en el desarrollo e integración nacional de cada una de ellas" (Fundación Frondizi, 2014, p. 35).

"Solo entonces, cuando la comunidad de la geografía, de la historia y del ideal se integren con la similitud del progreso económico-social y del desarrollo espiritual, sólo entonces cabrá afirmar la existencia de una cabal unidad continental" (Fundación Frondizi, 2014, p. 49).

Ejemplo práctico de esta visión de integración latinoamericana en su política exterior fue la reunión con Quadros, presidente brasileño. La relación con Brasil fue históricamente conflictiva, y especialmente en el contexto de Guerra Fría en nuestro continente, prevalecían las hipótesis de conflictos geopolíticos. Por tal razón, querer afianzar los lazos políticos con el país vecino denota una gran voluntad y visión de integración por parte de Frondizi.

La reunión celebrada en abril de 1961 marcó un gran acontecimiento en las relaciones entre Brasil y Argentina. La misma finalizó con la Declaración de Uruguaya, que puede interpretarse como un paso previo a la conformación del MERCOSUR (Llairó y Siepe, 2003, p. 171).

Otro destacado acontecimiento que refuerza la línea integracionista de su política exterior fue la firma del tratado de la Asociación Latinoamericana de Libre Comercio (ALALC) el 18 de febrero de 1960. Las negociaciones para la conformación de esta se iniciaron entre los países del Cono Sur, y Argentina formó parte de las mismas (ALADI, 2019).

La gran motivación que llevó a la creación de esta Asociación fue la de superar las dificultades estructurales a las que estaban supeditadas las economías regionales como, por ejemplo, la ya mencionada exportación de productos básicos. La firma de este tratado fue uno de los grandes pilares de política exterior frondicista en cuestiones latinoamericanas (Cardone, 2013).

Por otra parte, frente al caso de la revolución cubana, Frondizi continúo fiel a los principios que guiaban su política exterior, como el pragmatismo y la no intervención, lo que fue motivo de descontento y controversia, tanto a nivel interno como en la comunidad internacional.

Un claro ejemplo de las acciones que trajeron aparejado malestar fue la reunión del estadista Frondizi con el comandante Ernesto "Che" Guevara el 18 de agosto de 1961, a sabiendas de que Fidel Castro ya había proclamado el carácter socialista de la revolución. La intención era oficiar como interlocutor entre Estados Unidos y Cuba y demostrar la inconveniencia a este último sobre su ingreso al Pacto de Varsovia. Esto demuestra no sólo el carácter pragmático de su política exterior, sino también el fomento de la paz para evitar escaladas en conflictos, en este caso un conflicto regional (Alaniz, 2016).

Testimonio de Frondizi (citado en Menotti 1998): 
"Llevado a mi presencia, tuvimos una charla absolutamente a solas, que duró aproximadamente setenta minutos. Cuando abordamos el tema de la posibilidad de elaborar algún tipo de estatus de convivencia con Estados Unidos, me hizo notar que lo consideraba improbable por la resistencia de los grupos más duros del país del Norte; pero que, de todas maneras, si yo lo consideraba conveniente, explorara la posibilidad, pues él estaba de acuerdo en buscar, en la medida de lo posible, alguna salida al aislamiento".

Otro de los momentos culmines dentro de la política exterior frondizista fue la abstención del ministro de relaciones exteriores argentino, M. A. Cárcano, frente a la resolución sobre la exclusión cubana de la Organización de Estados Americanos (OEA). La misma tuvo lugar durante la octava conferencia ministerial que se llevó a cabo en Punta del Este (Uruguay), del 22 al 31 de enero de 1962. Aquí se observa perfectamente su postura anti ideológica y defensa del principio de no intervención (Bobadillo Gonzaléz, 2008).

Frente a tal hecho, el canciller argentino siguiendo las instrucciones de Frondizi, declaró en dicha Conferencia Ministerial lo siguiente:

"Queremos salvar la unidad del sistema interamericano y por ello nos abstendremos de votar sanciones que puedan vulnerar el principio de no intervención que irritan más las condiciones políticas actuales y que se prestarán a las actividades de los extremistas de izquierda y de derecha" (Cúneo, 1994, p.17).

\section{El Tratado Antártico, un gran acierto en la política exterior de Frondizi}

A fin de dar cuenta del último objetivo específico, es decir, el de explicar el contexto de la firma del Tratado Antártico, corresponde exponer que, previo a su firma, en el continente antártico se encontraban presentes los siguientes países que "mantuvieron los reclamos territoriales anteriormente avanzados" (Prieto Larrain, 2004, p. 141). Estos eran: Argentina, Australia, Chile, Francia, Nueva Zelanda, Noruega, el Reino Unido. Particularmente, la presencia de Argentina data desde el año 1904, cuando se izó por primera vez un pabellón argentino como prueba de presencia permanente. Desde ese entonces, primaba una política exterior de tipo unilateral con tintes nacionalistas, es decir, previo a la firma del Tratado, se tenía sobre la Antártida una mirada netamente nacionalista, debido a que la cuestión de la soberanía nacional era estrictamente primordial en dicho ámbito (Ministerio de Defensa, s/f).

A su vez, esta mirada nacionalista que regía por sobre la cuestión antártica, incidió en que en diferentes oportunidades la tensión se hiciera presente en la etapa anterior a la creación del Sistema Antártico. Claro ejemplo de ello, es la tensión existente entre Argentina, Chile y Gran Bretaña - tres países con pretensiones de soberanía-. Si bien habían firmado un acuerdo tripartito, la competencia entre ellos continuaba presente ${ }^{8}$, en palabras de Dodds (2009): "los tres países se ocupaban en crear cada vez más estaciones de investigación y buscaban marginalizar la presencia de los otros al destruir cualquier evidencia de ocupación anterior en el suelo" (p.37).

Por otra parte, en el contexto en el cual se arriba al Tratado, como se explicó con anterioridad, prevalecían los temas de seguridad y expansión geográfica debido al conflicto de Guerra Fría. Años previos a la firma, se cuestionaba en términos de seguridad qué pasaría con dicho continente y el futuro de la región. En 1956 India presenta el tema para ser tratado en la Asamblea General de Naciones Unidas por primera vez ${ }^{9}$, cuestionando las tensiones derivadas del continente antártico, y las problemáticas que esto podría traer como consecuencia en el corto

\footnotetext{
${ }^{8}$ En una ocasión Gran Bretaña envió buques de guerra como consecuencia de los disparos en forma de advertencia que británicos habían recibido por parte de Argentina. En otra oportunidad, las tensiones entre estos tres países se hicieron presentes cuando los británicos desmantelaron un refugio chileno en el territorio (Dodds, 2009).

9 "El gobierno de la India considera que, para poder fortalecer la paz universal, sería apropiado y oportuno para todas las naciones acordar y afirmar que el área será utilizada en su totalidad con fines pacíficos y para el bienestar general" (Dodds, 2009, p. 37).
} 
plazo, sobre todo si se tenía en cuenta el contexto internacional que transcurría en ese momento, en donde la lógica de balanza de poder permanecía intacta. Dicho planteo trajo aparejadas tensiones entre los países reclamantes de soberanía, dado que se mostraban contrarios a la posición de India. No obstante, permitió que la cuestión de la Antártida pudiera pensarse y abordarse en forma colectiva.

Tal es así que, en pleno auge de la división del mundo entre este-oeste, se lleva a cabo la celebración del Año Geofísico Internacional, que tuvo lugar en el periodo de 1957-1958, y de la cual participaron más de treinta mil (30.000) científicos y 66 países $^{10}$, incluyendo a la Argentina. Durante ese año se realizaron investigaciones físicas y químicas con el objetivo de estudiar la Tierra y la atmósfera. El tema de la Antártida no fue ajeno a las mismas y despertó un gran interés entre los países participantes (Colacrai, 2001, p.17).

A raíz de esto, Estados Unidos convoca a aquellos países que habían participado del Año Geofísico, a una Conferencia en Washington en el año 1959 con el objetivo de debatir la cuestión Antártida. Argentina envía representantes, participando tal como lo hizo dos años anteriores (Colacrai, 2001).

Los grandes debates que se llevaron a cabo sobre la Antártida fueron la necesidad de cooperación científica en el área, el equilibrio pacífico y la no militarización, y la desnuclearización. Estos temas que abogaban por la cooperación internacional, son un punto a destacar, especialmente, teniendo en cuenta el contexto internacional en el que se debatió.

Era inminente que, como producto del aumento de la interdependencia entre las diferentes naciones, se llegara como consecuencia a un incremento de la cooperación entre ellas.

Como resultado de dicha Conferencia, se firmó el 1 de diciembre de 1959 el Tratado Antártico, con el propósito de dar al continente un status jurídico adecuado, con marco legal propio para la protección de los intereses de la región. Allí se inicia la configuración del hoy conocido Sistema del Tratado Antártico, conformado por el Tratado principal y una serie de acuerdos relacionados con la temática que se firmaron con posterioridad. El mismo entró en vigencia el 23 de junio de 1961 (Ministerio de Relaciones Exteriores, Comercio Internacional y Culto, s/f).

La cooperación internacional pacífica fue sin dudas el eje de mayor relevancia que se plasmó en dicho acuerdo. Muestra de ello, es uno de los primeros párrafos redactados en el Tratado: "Reconociendo que es en interés de toda la humanidad que la Antártida continúe utilizándose siempre exclusivamente para fines pacíficos y que no llegue a ser escenario u objeto de discordia internacional" (Tratado Antártico, 1959, p.1).

Por otra parte, al enviar Arturo Frondizi un representante a la Conferencia de Washington, firmando y adhiriendo al Tratado, pasó a ser en uno de los miembros fundadores del régimen. Al momento de firmar, Argentina continúa preservando, al igual que los otros Estados, la condición de país reclamante de soberanía en el territorio continental antártico, pero la misma se combina con la cooperación internacional prevaleciente del momento.

La visión internacional del presidente era la de que el mundo marchaba hacia la distensión, hacia el desarme general, y afirmaba que Argentina debía contribuir a la idea de consolidación de la paz mundial. Esto formaba parte del "Mundo Uno" que el mandatario entendía que era el futuro, con un escenario internacional completamente globalizado y desnuclearizado, en donde primara la cooperación entre los Estados, y por tal motivo, la adhesión al Tratado puede ser entendida como ícono dentro de su política exterior.

Este pensamiento se plasma en el artículo V del Tratado: "Toda explosión nuclear en la Antártida y la eliminación de desechos radiactivos en dicha región quedan prohibidas" (Ministerio de Defensa, s/f). Argentina fue quien propuso la incorporación del mismo, ya que alegaba que

${ }^{10}$ Entre estos se encontraba la Unión Soviética. Esta participación en el programa de investigación causó cierto resquemor; muchos países temían que el comunismo internacional quedara en el continente una vez que las investigaciones finalizaran (Dodds, 2009). 
durante las sesiones de la Conferencia, jamás se había planteado esta cuestión, y, si no se incorporaba habría una plena libertad para realizar todo tipo de explosiones nucleares en la Antártida, y la misma quedaría como un espacio de depósito de materiales radioactivos.

A partir de la propuesta de la delegación argentina surgió un debate entre quienes estaban a favor de la prohibición total de todo tipo de explosiones, como por ejemplo Argentina y la Unión Soviética, y por otro lado, quienes pretendían una libertad condicionada. Si bien en un momento gran parte de las delegaciones se mostraron contrarias a la incorporación propuesta por Argentina, la misma terminó triunfando, y se cristalizó en el artículo $\mathrm{V}$ del Tratado.

De allí deriva la cabal importancia que Frondizi le otorga a este Tratado, ya que, tal como Colacrai (2001) afirma,

La defensa del Tratado Antártico como un aporte a la paz y concordia internacional se desprende de la propia cosmovisión profesada por el desarrollismo y de sus percepciones acerca de la gestación de un nuevo modelo de las relaciones internacionales signado por "coexistencia pacífica" (p. 56).

Empero, este acontecimiento no fue muy bien recibido en algunos sectores políticos, lo que derivó en un debate parlamentario para la posible ratificación del Tratado. Si bien en Senadores el debate se caracterizó por ser más sencillo ${ }^{11}$, no fue así en la Cámara de Diputados de la Nación Argentina, ya que legisladores, especialmente aquellos provenientes de la UCRP, veían a la ratificación del Tratado como una renuncia a la soberanía argentina sobre el continente antártico, y no como un "stand by" o "congelamiento" de la misma, tal como lo entendía la UCRI con A. Frondizi. El debate dejó en evidencia las diferencias entre los partidos políticos.

Aquellos que se oponían, no sólo "emplearon argumentos relacionados con el desmembramiento territorial y la pérdida de la soberanía", hasta términos muy duros como "acto de renunciamiento" y "capitulación" (Colacrai, 1997, p. 214), sino que también, se mostraban en contra de la posición tomada por las fuerzas armadas argentinas, sosteniendo que se equivocaban en apoyar la firma del Tratado, ya que su función era la de proteger y preservar la soberanía e integridad del territorio nacional, y que el reclamo territorial de Argentina en el continente antártico formaba parte de dicha tarea de defensa ${ }^{12}$.

Sin embargo, la discusión sobre los posibles efectos que el Tratado podría tener sobre la soberanía argentina en la región, tenía de trasfondo una diferencia tajante sobre la autopercepción de la posición del país en el mundo. Mientras que el presidente junto a su partido sostenía que la participación del régimen era una excelente forma de inserción internacional, y de ser partícipes de la cooperación, sus férreos oponentes defendían la no participación en bloques de índole político, económico o militares, y, a su vez, sostenían que la ratificación sólo beneficiaría a las grandes potencias del momento, Estados Unidos y la Unión Soviética, dejando entrada libre a esta última en el continente (Colacrai, 2001).

Esta situación particular se puede analizar, nuevamente, retomando el concepto de diplomacia de doble filo de R. Putnam, la cual fue explicada en párrafos anteriores. Por un lado, en la "mesa internacional" en Washington, se negoció con el objetivo de maximizar los beneficios que el gobierno de Frondizi pretendía obtener con la firma del Tratado, mientras que los otros Estados partícipes pretendían lograr lo mismo para sus respectivos intereses. Mientras que en la "mesa nacional" debía responder ante las demandas del Congreso y, a su vez, negociar con este para la posible ratificación.

\footnotetext{
11 "En Senadores tuvo un trámite más rápido y sencillo, aconsejándose su aprobación, a partir del cual se remitió en septiembre de 1960, para su tratamiento en Diputados" (Colacrai, 2001, p. 50).

12 Por ejemplo, el diputado Cuevaz argumentaba lo siguiente “...es increíble que a esta altura de nuestra historia debamos asumir la defensa de nuestros derechos efectivos en la Antártida frente a la amenaza que se cierne sobre ella si se aprueba la ratificación de este tratado. Es lamentable que aún debamos discutir si tenemos que defender nuestra soberanía sobre la Antártida, porque en esencia ese es el trasfondo del problema..." (Diario de Sesiones de la Cámara de Diputados en Colacrai, 2001, p. 51).
} 
Sin embargo, no hubo solamente desacuerdos frente a tal hecho, sino que para muchos sectores políticos de la sociedad "el país recibía por primera vez reconocimiento de potencia antártica" (Colacrai, 1997, p. 214). Y, pesar de esta notable oposición que el entonces presidente recibió por su deseo de formar parte de este nuevo régimen, "la Argentina firmó y la historia le dio la razón al presidente Frondizi" (Rinaldi, 2013, p. 153), debido a que el Sistema Antártico logró mantenerse en vigencia a través del tiempo, desde el momento de su creación.

Entre las actividades realizadas en la Antártida, luego de la firma del Tratado que dio origen a dicho Sistema, se destaca principalmente, el viaje que realizó Frondizi en 1961, el año de la ratificación, al continente, llegando a la base de la Antártida como una forma de ratificar la soberanía argentina sobre el territorio, y para evitar especulaciones en sus oponentes sobre la traición a la soberanía del país en dicha región.

Otras tareas relevantes que se llevaron a cabo en el período 1959-1962 fueron las siguientes:

- Se desarrollaron trabajos hidrográficos, oceanográficos, biológicos de determinación de la convergencia antártica, aerofotogramétricos, de balizamiento, meteorología y glaciología.

- Actividades científicas a cargo del Instituto Antártico Argentino en biología, fisiología humana, geología y oceanografía.

- En el primer vuelo al Polo Sur durante la campaña de 1961-62, el Cap. Quijada realizó la proeza de unir los dos continentes, con aviones Douglas DC-3, versión C-47 de la Aviación Naval, matriculados como el CTA-15 y el CTA-12.

- Cuatro veces flameó en el Polo Sur la bandera argentina. Los hombres de la Armada la izaron el 6 de enero de 1962; los de la Fuerza Aérea, el 3 de noviembre de 1965; los del Ejército, el 10 de diciembre del mismo año, y la Expedición Milenio, el 5 enero del 2000 (Rinaldi, 2013, p. 153).

En consonancia respecto a lo que se fue argumentando a lo largo del presente trabajo en lo relativo al pensamiento desarrollista con el consecuente pragmatismo en su política exterior, al Tratado Antártico y su defensa se lo puede entender como "un aporte a la paz y concordia internacional [que] se desprende de la propia cosmovisión profesada por el desarrollismo y de sus percepciones acerca de la gestación de un nuevo modelo de las relaciones internacionales signado por la 'coexistencia pacífica'”(Colacrai, 1997, p. 215).

\section{Conclusiones}

La llegada de Frondizi a la presidencia sin dudas supuso un cambio cualitativo, tanto por el modelo desarrollista impulsado a nivel interno, como también por su pragmática, y un tanto cosmopolita visión del mundo plasmada en su política exterior.

En cuanto a su gestión en el contexto interno, entendía al modelo desarrollista como un plan de políticas a seguir a largo plazo, y no como un esquema meramente coyuntural. Argumentaba que sólo a través de esta línea, Argentina iba a poder desarrollarse e insertarse en el mundo que él percibía como globalizado, y que, para lograr tal objetivo, era necesario cerrar las puertas al ingreso de manufacturas y combustibles, para abrírselas al capital extranjero, a fin de producir dentro de nuestras fronteras los mismos tipos de manufactura, tanto de origen agropecuario, como industrial.

No obstante, diferentes sectores sociales no supieron acompañar y comprender este modelo de sustitución compleja de importaciones. La importación de nuevas tecnologías, como la llegada masiva de inversiones extranjeras directas al país, supusieron para muchos una traición a la patria y a la causa nacional de desarrollo. Claro ejemplo de ello fue el cuestionamiento al asunto petrolero en ese entonces y las disputas que ello supuso.

Asimismo, el tinte pragmático en la política exterior de Arturo Frondizi fue necesario para poder implementar el modelo desarrollista en nuestro país en un contexto signado por la Guerra Fría, con lo que ello suponía. Mantener relaciones diplomáticas y comerciales con 
diferentes Estados, en función si era favorable para Argentina o no, y dejando de lado la distinción ideológica que separaba al mundo en ese entonces, era esencial para poder obtener todos los beneficios necesarios a fin de impulsar el desarrollo en nuestro país. Indudablemente, según la visión del mandatario, los beneficios económicos se verían plasmados en Argentina, así se mantuviera relaciones con un país bajo la órbita del comunismo o capitalismo.

Además, como gran visionario que fue, entendía que el conflicto este-oeste llegaría a su fin, abriendo paso a un "Mundo Uno", en donde primaría la cooperación entre los Estados y la paz a nivel mundial, en un mundo completamente globalizado. Por tal motivo, Frondizi afirmaba que Argentina debía prepararse y obtener el máximo beneficio de la misma, pensando en el modelo desarrollista que impulsaría e insertaría a nivel mundial a nuestro país.

Por otra parte, la firma del Tratado Antártico (1959) y su ratificación en 1961 son un ejemplo íntegro de su visión que prioriza la coexistencia pacífica, y a su vez, es rotundamente un componente de su genuina visión para llevar a la práctica su idea de "Mundo Uno", desarmado, no nuclear e integrado. Allí se acuerda la cooperación internacional entre los países, a pesar de las diferencias ideológicas existentes (cabe recordar que, dentro del Sistema Antártico, Estados Unidos y la entonces Unión Soviética, también son miembros fundadores).

El Tratado prefigura al mundo que se aproximaba, un mundo globalizado, en donde las relaciones multilaterales y la cooperación son vías esenciales. Su ratificación es un icono dentro de la política exterior de Frondizi, considerado la máxima expresión de su visión internacional, más allá de lo que representa este Tratado en el contexto internacional en que se firma.

A su vez, su firma con el consecuente objetivo de cooperar entre países y mantener a la región como zona de paz, desnuclearizada y propicia para la investigación, forma parte de los principios de la política exterior del entonces presidente argentino, ya que la persecución de la paz era un principio clave de su política exterior. Ser uno de los precursores del Sistema Antártico fue un claro reflejo de su visión internacional, un tanto cuestionada por muchos sectores, que, sin lugar a duda, contribuyó a su fatídico desenlace en la presidencia argentina. Claro ejemplo de ello fue la discusión en Diputados para la ratificación del Tratado Antártico.

Pese a las numerosas críticas que tuvo a lo largo de su mandato presidencial, Frondizi podría ser considerado un buen estadista. Este intelectual, impulsor del desarrollismo en la Argentina, probablemente ejerció la presidencia en el momento y tiempo equivocados; su visión acerca del modelo que debía llevar el país y de cómo debían ser las relaciones internacionales y la política exterior argentina, no pudo ser ni comprendida ni aceptada por muchos, tanto a nivel interno como internacional.

Esta falta de comprensión, sumada a la presión interna por el temor al peronismo, y a nivel internacional por miedo al comunismo, y teniendo en cuenta la firmeza que Frondizi tenía en sus convicciones y en lo que era correcto para el país, fueron las causas de su posterior y adelantado derrocamiento, ya que no pudo finalizar el periodo de su mandato presidencial.

En conclusión, siguiendo las palabras de Di Tella (1982), "el gobierno de Frondizi marcó un cambio cualitativo en la marcha del país, fue una especie de aggiornamento integral al cual el país se lanzó".

\section{Referencias bibliográficas}

Alaniz, R. (2016). Visión Desarrollista, Visión Desarrollista. 11 de diciembre. Recuperado de: http://www.visiondesarrollista.org/frondizi-che-puertas-cerradas/ . Consulta: 22 de marzo de 2020

Blanco, G. Á. (2015). El gobierno desarrollista de Arturo Frondizi analizado desde la óptica del estructuralismo de Marcelo Diamand. Perspectivas, 5 (1), pp. 13-34 
Bobadilla González, L. (2006). La exclusión de Cuba de la Organización de los Estados Americanos: los desacuerdos diplomáticos entre México y Estados Unidos en 1962. Dirección General del Acervo Histórico Diplomático-Secretaría de Relaciones Exteriores, pp. 58-76

Cardone, E. H. (2013). Porque Frondizi fue un estadista. Buenos Aires: Eder

Carsen, M. V. (2017). El encuentro de Frondizi-Franco y el desdibujamiento de diferencias ideológicas para la promoción de desarrollo. RES GESTA, 53, pp. 1-19

Casa Rosada. Presidencia (s/f). Arturo Frondizi (1958-1962). Recuperado de: https://www.casarosada.gob.ar/informacion/discursos/18-nuestro-pais/galeria-depresidentes/437-arturo-frondizi-1958-1962. Consulta: 20 de noviembre de 2019

CEPAL (2016). Desarrollo e Integración en América Latina. Washington: CEPAL

Cisneros, A. (2016). La política exterior de Frondizi. Visión Desarrollista. Recuperado de: http://www.visiondesarrollista.org/2571-2/. Consulta: 23 de diciembre de 2019

Colacrai, M. (1997). Cambios y continuidades en la política antártica argentina, 1959-1983. Ciclos, 12, pp. 203-221

Colacrai, M. (2001). Continuidades y cambios en la política Antártica Argentina 1958-2001. Rosario: Editorial Académica Española

Cúneo, D. (1994). La política internacional del presidente Frondizi y la crisis del subdesarrollo: Punta del Este, Cuba y Uruguayana. En Pisarello Virasoro y Menotti, E. Arturo Frondizi. Historia y Problemática de un estadista. Buenos Aires: Depalma

De Pablo, J. C. (1998). Frondizi-“Nomia”, 40 años después. Contexto. Suplemento a la entrega $n^{\circ} 450$, pp. $1-24$

Devoto, L. M., \& Pelosi, H. C. (2009). Frondizi y Eisenhower, ¿relación amistosa o incierta? Temas de historia argentina y americana, 14, pp. 56-93

Devoto, L. M. (2010). Viaje del presidente Frondizi a Canadá y Asia. Temas de historia argentina y americana, 16, pp. 147-176

Dodds, K. (2009). La administración del continente polar: los orígenes geopolíticos del Tratado Antártico de 1959. Revista de Historia Internacional, 39, pp. 27-49

Espacio Radical (s/f). Términos políticos y otros. UCRI-UCRP. Recuperado de: http://www.espacioradical.com.ar/index.php/enciclopedia/Enciclopedia-1/U/UCRIUCRP-31/. Consulta: 22 de noviembre de 2019

Fundación Frondizi (2012). Arturo Frondizi, mensajes presidenciales 1958 -1962. Ciudad Autónoma de Buenos Aires: blancas.indd

García Bossio, H. (2012). Pensamiento y praxis de Rogelio Frigerio, fundador del proyecto desarrollista en Argentina. Tesis de Doctorado en Ciencias Políticas, Universidad Católica Argentina, Facultad de Ciencias Sociales, Políticas y de la Comunicación, Instituto de Ciencias Políticas y Relaciones Internacionales. Recuperado de: http://bibliotecadigital.uca.edu.ar/repositorio/tesis/pensamiento-praxis-rogeliofrigerio.pdf. Consulta: 15 de agosto de 2019

Lanús, J. A. (2000). De Chapultepec al Beagle. Buenos Aires: Emencé Editores S.A.

Leal Buitrago, F. (2003). La doctrina de seguridad nacional: materialización de la Guerra Fría en América del Sur. Revista de Estudios Sociales, 15, pp. 74-87

Llairó, M. M. y Siepe, R. (2003). Frondizi: un nuevo modelo de inserción internacional. Ciudad de Buenos Aires: EUDEBA

Machinandiarena de Devoto, L. M. (2015). Visita del presidente Arturo Frondizi al Reino Unido. Investigaciones y Ensayos, 61, pp. 201-248 
Malatesta, A. A. (2014. El desarrollismo argentino y su tiempo. Proyecto de investigación, Córdoba (San Francisco): Departamento de Investigación UCES

Marcial, M. M. (1961). El Tratado Antártico. Anales de la Universidad de Chile, pp. 179-192.

McMahon, R. J. (2009). La Guerra Fría. Una breve introducción. Madrid: Alianza Editorial

Menotti, E. (1998). Testimonio de Arturo Frondizi. Buenos Aires: Editorial Planeta

Ministerio de Defensa (s/f). Antártida. Historia. Recuperado de: https://www.argentina.gob.ar/armada/antartida/historia . Consulta: 25 de enero de 2020

Ministerio de Relaciones Exteriores, Comercio Internacional y Culto (s/f). Tratado Antártico. Recuperado de: https://cancilleria.gob.ar/es/iniciativas/dna/divulgacion/tratadoantartico. Consulta: 15 de noviembre de 2019

Morgenfeld, L. (2011-2012). Desarrollismo, Alianza para el Progreso y Revolución Cubana. Frondizi, Kennedy y el "Che" en Punta del Este (1961-1962), en Ciclos, 20 (39-40), pp. $133-163$

Neyra, H. E. (2017). El desarrollismo en Argentina: la experiencia frondicista (1958-1962). Textos para Discussão PPGE/UFRGS, 4, pp. 1-17

Organización Internacional del Trabajo (s/f). Convenio de migración entre la República Argentina $y$ el Estado Español. Recuperado de: https://www.ilo.org/dyn/natlex/natlex4.detail?p_lang=es\&p_isn=38910\&p_country=A RG\&p_count $=1355$. Consulta: 25 de marzo de 2020

Pelosi, H. C. y Machinandiarena de Devoto, L. (2012). Las relaciones internacionales en la presidencia de Frondizi 1958-1963: desarrollo, integración latinoamericana y paz mundial. Saarbrücken: Editorial Académica Española

Pontoriero, E. D. (2015). Estado de excepción y contrainsurgencia: el Plan Conintes y la militarización de la seguridad interna en la Argentina (1958-1962). Contenciosa, 4, pp. $1-17$

Prieto Larrain, M. C. (2004). El Tratado Antártico, vehículo de paz en un campo minado. Universum, 19 (1), pp. 138-147

Putnam, R. (1988). Diplomacy and Domestic Politics: The Logic of Two-Level Games. International Organization, 42 (3), pp. 427-460

Rinaldi, C. A. (2013). Desarrollo Científico Argentino en la Antártida. Boletín del Centro Naval, 836, mayo-agosto, pp. 147-156

Szusterman, C. (1993). Frondizi and the Politics of Developmentalism in Argentina, 1955-62. London: Palgrave Macmillan UK

Tratado Antártico. Washington, $1^{\circ}$ de diciembre de 1959. Recuperado de: https://documents.ats.aq/keydocs/vol 1/vol1 2 AT Antarctic Treaty s.pdf. Consulta: 23 de enero de 2020

Tratado de Montevideo. Tratado que establece una zona de libre comercio e instituye la Asociación Latinoamericana de Libre Comercio. Montevideo, 18 de febrero de 1960. Recuperado de http://www.aladi.org/sitioaladi/?page_id=4798. Consulta: 20 de diciembre de 2019

Zapata, V. (2005). Revisión de Ezquerro, M. L. (2005). La política exterior de Arturo Frondizi 1958-1962, Centro de Estudios Avanzados, Córdoba. Recuperado de: http://sedici.unlp.edu.ar/handle/10915/9804. Consulta: 15 de noviembre de 2019 